Wiaam M AL-Ashou BDS, M.Sc (Asst Lec)

\section{The Effect of Various Bur Types and Two Bonding Agents on Shear Bond Strength of Composite Resin to Dentin}

Dept of Operative Dentistry

College of Dentistry, University of Mosul

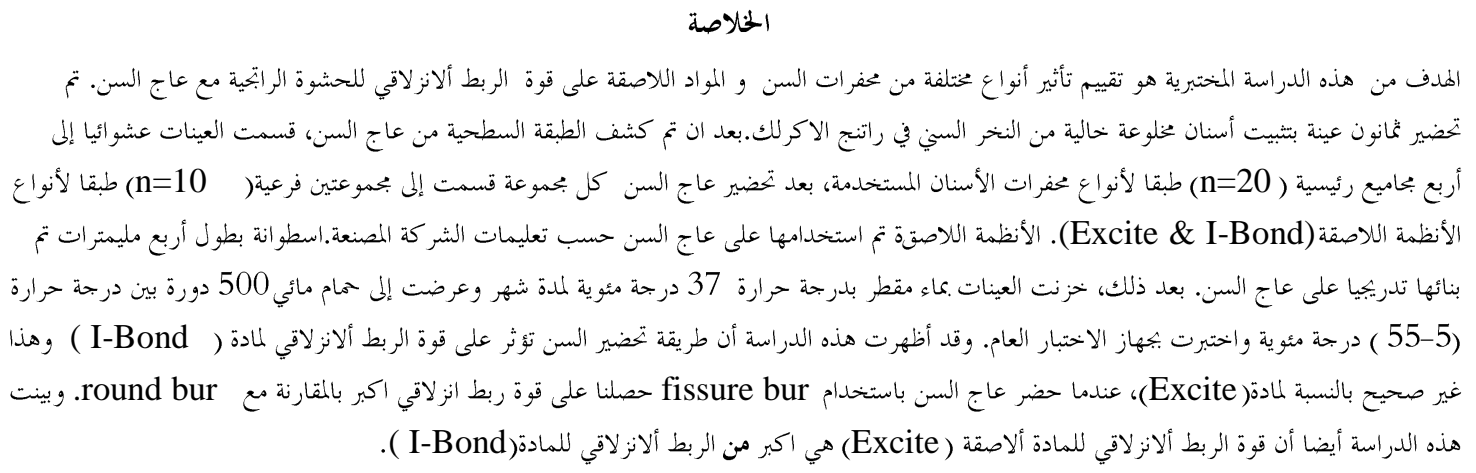

\begin{abstract}
Aims: The aims of this in vitro study was to assess the effectiveness of various bur types and bonding agents on the shear bond strength of composite resin to dentin. Eighty non carious, extracted human molars were mounted in acrylic resin, the occlusal surface of all the teeth were grounded to obtain flat dentin surface. After that the teeth were randomly divided into four groups $(n=20)$ according to the bur types that will be used to prepare the dentin surface. After preparation of dentin each group was divided into two subgroups $(\mathrm{n}=10)$ according to the type of adhesive systems used (Excite \& I-Bond), the adhesive systems were applied to the dentin surface and light-cured according to manufacturers instructions. A $4 \mathrm{~mm}$ high composite cylinder was incrementally built up. After that, specimens stored for one month at $37^{\circ} \mathrm{c}$ in distilled water and thermo cycled for 500 cycles between $\left(5^{\circ} \mathrm{c} \& 55^{\circ} \mathrm{c}\right)$ and then tested in a universal testing machine. The results of this study demonstrated that the method of surface preparation affected the shear bond strength of I-Bond and this is not true for Exit system, when the dentin surface prepared with fissure burs the shear bond strength greater than when the surface prepared with round burs. The results of this study also showed that the groups in which Excite bonding agent used have greater value of shear bond than the groups treated with I-Bond.

Key words: Dental burs, bonding agents, shear bond, composite resin.

AL-Ashou WM. The Effect Of Various Bur Types and Two Bonding Agents on Shear Bond Strength of Composite Resin to Dentin. Al-Rafidain Dent J. 2012; 12(2): 242-248.

Received: 5/4/2010 Sent to Referees: 5/4/2010

Accepted for Publication: 27/3/2011
\end{abstract}

\section{INTRODUCTION}

During cavity preparation, tooth structure cut by rotary or manual instruments and a large amount of energy is generated locally, which result in denaturated collagen, and lead to chemical and physical alteration in the surfaces of the tooth. This procedures produces smear layer, which can influence the adhesion between the tooth structure and dental materials. It has been report that there is a micromorphologic difference between dentin cut by diamond and carbide rotary instrument. The surface cut with diamond burs examined by scanning electron microscope present more irregularities and thicker smear layer when compared to the dentin surface cut with carbide burs. ${ }^{(1,2)}$

Dentin bonding systems have been improved in order to promote durability and reliability of adhesive restorations ${ }^{(3)}$. The mechanisms of adhesion of currently used adhesive, depending on clinical approach to the smear layer: it can be removed by acids (the fourth generation and the fifth generation) or dissolved by self etching primers(the sixth generation and seventh generation).$^{(4)}$ 
Most of dentin bond strength studies are performed on specimens that have been ground with 600-grit silicon carbide paper. However, adhesive resins are clinically applied on dentin prepared by bur fixed in an air turbine or a high-speed hand piece.

The aim of this study to evaluate the effect of different bur types (diamond round bur, diamond fissure bur, carbide round bur, and carbide fissure bur) and two types of bonding agent Excite (fifth generation ) and I-Bond (seventh generation) on shear bond strength of composite resin to dentin.

\section{MATERIALS AND METHODS}

Eighty extracted human non carious molars were used in this study. Routine prophylactic procedure was carried out with rubber cup and aqueous slurry of pumice for all teeth, after cleaning all teeth underwent careful microscopic examination((Motic, TAIWAN) in order to exclude those with visually undetectable cracks before they were treated. Samples were mounted vertically on acrylic resin block. To obtain flat dentin surfaces at depth of $1.5 \mathrm{~mm}$ the occlusal surface of all teeth were ground with using a diamond wheel saw (KG Sorensen SP, Brazil) under water coolant where a groove market at $1.5 \mathrm{~mm}$ served as standardization. Then, the flat dentin surface abraded with 400, 600 grit wet silicon carbide papers mounted on a grinding machine withcopious amounts of water to create standardized dentin surface. ${ }^{(1,5,6)}$ After that the teeth were divided according to the type of bur that use to prepare the dentin surface into four groups $(n=20)$ as follow:

Group1: the dentin surface ground with diamond round bursNo.1014KG Sorensen Ind com. Ltd., Brazil) using

high-speed handpiece with air-water spray, in such a way the long axis of the bur was perpendicular to the dentin surface.

Group2: the dentin surface ground using a diamond straight fissure burs No.1092 (KG Sorensen Ind com. Ltd., Brazil) using high-speed handpiece with air-water spray, in such a way the long axis of the bur was parallel to the dentin surface.

Group3: the dentin surface ground using a tungsten carbide round burs No.3 (Mani Inc. Japan) using high-speed handpiece with air-water spray, in such a way the long axis of the bur was perpendicular to the dentin surface.

Group4: the dentin surface ground using a tungsten carbide straight fissure burs No.56 (Mani Inc. Japan) using high-speed handpiece with air-water spray, in such a way the long axis of the bur was parallel to the dentin surface.

In each group following dentin preparation, the area of bonding was defined using circular perforation of a self adhesive tape measuring $4 \mathrm{~mm}$ in diameter so that the applied adhesive agents confined to a standardized area on the base. The specimens in each group were randomly divided into two subgroup $(\mathrm{n}=10)$ according to the type of adhesive system used as follows:

Group I: each prepared dentin surface was etch with $37 \%$ phosphoric acid gel (Vivdent Ets, Schaan/Liechtenstein, Germany) for 15 seconds, rinsed for 20 seconds, then gently blown to remove excess water (maintain a moist surface), a total etch adhesive system (Excite, Ivoclar, Vivadent, Liechtenstein) was applied according to the manufacturers instructions.

Group II: a one-step self-etch adhesive system (I- Bond, Medicinos Linija UAB., EU) was applied on dentin surfaces according to the manufactures instructions. The composition of Excite and I-Bond seen in (Table 1).

Table (1) The composition of the bonding systems used in the study

\begin{tabular}{ll}
\hline Bonding system & Composition \\
\hline Excite (total etch) & HEMA, TEGDMA, Phosphoric acid 37\%, acrylate, Silicon \\
& dioxide, ethanol \\
I-Bond(self etch) & UDMA, 4 Met, acetone, water, glutaraldhyde, camphorqui- \\
& none alcohol \\
\hline
\end{tabular}


Rubber mold with 4 x $4 \mathrm{~mm}$ dimension central hole was applied over the adhesive tape that was placed over the dentin surface base so that the hole in the rubber mold was positioned over the hole in the adhesive tape and attached in it is positions by two points of wax to the acrylic block. The mold was split vertically in one place through its entire thickness using a surgical blade No.23 by so doing, its later removal from around the composite was facilitated without putting undue stress on the composite sample. Subsequently, Tetric ceram composite (Ivoclar, Vivadent, Liechtenstein) shade A2 was packed directly against the demarcated dentin surface through the rubber hole with ash plastic instrument and adapted to avoid air entrapment in a two increments $2 \mathrm{~mm}$ thickness of each increment, according to manufacturer's instructions, after the application of first increment the thickness of the increment was checked by using grad- uate periodontal probe, then light polymerized for 40 second at $400 \mathrm{~mW} / \mathrm{cm}^{2}$ using a Quartz-Tungsten-Halogen (QTH) light curing unit (Astralis 5, Vivadent, Schaan/Liechenstein, Germany), the radiometer was used to check the light efficiency before starting each restoration, then the second increment was applied and covered with a transparent celluloid strip and light cure for 40 second. To standardized the curing distance the tip of the polymerization unit was applied in contact with the surface of the rubber mold. After that the wax points were removed and the composite post were released from the rubber mold and the adhesive tape, then each composite post polymerized for 10 second at four point all around to ensure that there was complete polymerization, thus create a cylinder of composite $4 \mathrm{~mm}$ in diameter and $4 \mathrm{~mm}$ in height bonded to the dentin surface at $90^{\circ}$ angle (Figure 1).

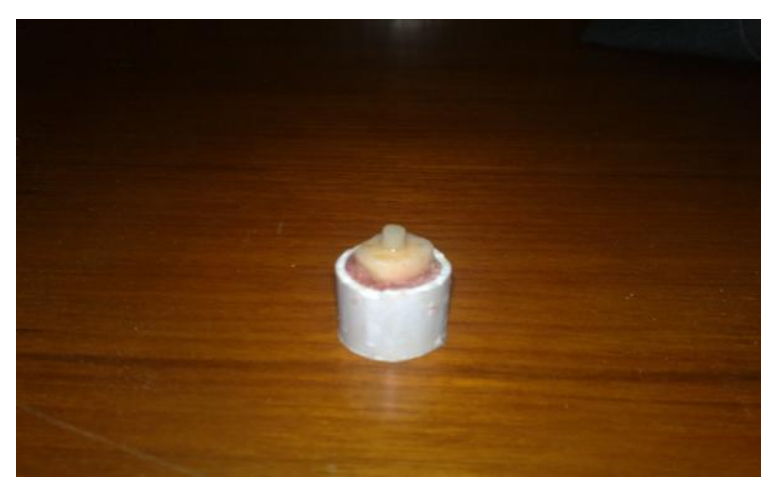

Figure (1) The tooth mounted in polyvinyl plastic ring and the tooth builds up with composite core.

All specimens were incubated inside an incubator (Fisher Scientific, Germany) for 30 day at $37^{\circ}$ in distilled water. Shear bond strength was evaluated using universal testing machine with knife- edged rod of $0.5 \mathrm{~mm}$ width at across head speed of $1.0 \mathrm{~mm} / \mathrm{min}$ that applied to dentin composite interface the bond strength and failure was calculated as the failure load divided by the surface area $\left(12.56 \mathrm{~mm}^{2}\right)$ and express in megapascals (Mpa), the data were calculated and analyzed statistically using unpaired T-test and Duncan's multiple range test at $(p<0.05)$.

\section{RESULTS}

Mean shear bond strength of composite resin to dentin after treated with different bur ranged from $(33.83 \pm 4.58) \mathrm{MPA}$ to $(36 \pm 3.39)$ MPA for the total etch groups, and from $(19.67 \pm 0.5)$ MPA to (29.86 \pm 3.97$)$ MPA for the self etch groups as shown in table (2 and 3 ). 
Table (2): Duncan's Multiple Range Test for the effect of bur types on the shear bond strength of composite resin to dentin when use Excite bonding agent.

\begin{tabular}{clccc}
\hline Group & Number & Mean & $\mathbf{\pm S D}$ & Duncan's Grouping \\
\hline G1,I & 10 & 33.83 & 4.58 & $\mathbf{A}$ \\
G2,I & 10 & 35.26 & 1.04 & $\mathbf{A}$ \\
G3,I & 10 & 34.11 & 4.58 & $\mathbf{A}$ \\
G4,I & 10 & 36.33 & 3.39 & $\mathbf{A}$ \\
\hline
\end{tabular}

G1=Diamond round bur G2=Diamond fissure bur G3=Carbide round bur $\quad$ G4=Carbide fissure bur $\mathrm{I}=$ Excite

Table (3): Duncan's Multiple Range Test for the effect of bur types on the shear bond strength of composite resin to dentin when use I-Bond.

\begin{tabular}{ccccc}
\hline Group & Number & Mean & 土SD & Duncan's Grouping \\
\hline G1,II & 10 & 19.67 & 0.50 & $\mathbf{C}$ \\
G2,II & 10 & 25.70 & 2.00 & $\mathbf{B}$ \\
G3,II & 10 & 20.67 & 1.32 & $\mathbf{C}$ \\
G4,II & 10 & 29.86 & 3.97 & $\mathbf{A}$ \\
\hline Diamond round bur & G2=Diamond fissure bur & G3=Carbide round bur & G4=Carbide fissure \\
II=I-Bond & & & &
\end{tabular}

Analysis of the data showed that the types of bur not significantly affect the shear bond strength of composite resin to dentin when total etch adhesive system (Excite) were used.

While for self etch group the types of bur significantly affect the shear bond strength of composite resin to dentin at $(p<0.05)$. When fissure burs used for the preparation the bond strength was greater than that obtained when dentin surface prepared with round burs. The result of this study also showed that the group that treated with carbide fissure bur produce higher bond strength than diamond fissure bur ( Table 3).

The means of shear bond strength for the groups treated with total etch adhesive(Excite) is greater than the groups treated with Self etch adhesive (I-Bond) as shown in Table (4).

Table (4): Duncan's multiple Range Test for the effect of different bur types and two bonding on the shear bond strength of composite resin to dentin.

\begin{tabular}{rcccc}
\hline Group & Number & Mean & $\mathbf{\pm S D}$ & Duncan's Grouping \\
\hline G1,I & 10 & 33.83 & 4.58 & $\mathbf{A}$ \\
G2,I & 10 & 35.26 & 1.04 & $\mathbf{A}$ \\
G3,I & 10 & 34.11 & 4.58 & $\mathbf{A}$ \\
G4,I & 10 & 36.33 & 3.39 & $\mathbf{A}$ \\
G1,II & 10 & 19.67 & 0.50 & $\mathbf{D}$ \\
G2,II & 10 & 25.70 & 2.00 & $\mathbf{C}$ \\
G3,II & 10 & 20.67 & 1.32 & D \\
G4,II & 10 & 29.86 & 3.97 & B \\
\hline
\end{tabular}

G1=Diamond round bur G2=Diamond fissure bur G3=Carbide round bur G4=Carbide fissure bur $\mathrm{I}=$ Excite $\quad \mathrm{II}=\mathrm{I}-\mathrm{B}$ ond

Microscopic examination using a stereomicroscope (Hamilton by AITAY International Italy) at a magnification level of X 20 of the prepared dentin surfaces with different bur types seen in Figure (2). The microscopic examination of debonded surfaces exhibited mixed failure
Figure (3) (adhesive +cohesive within adhesive resin) with few bonding remaining on the dentin surface for the groups treated with carbide fissure bur and diamond bur using with Excite bonding agent while other groups show mainly adhesive failure (Table 5). 
a
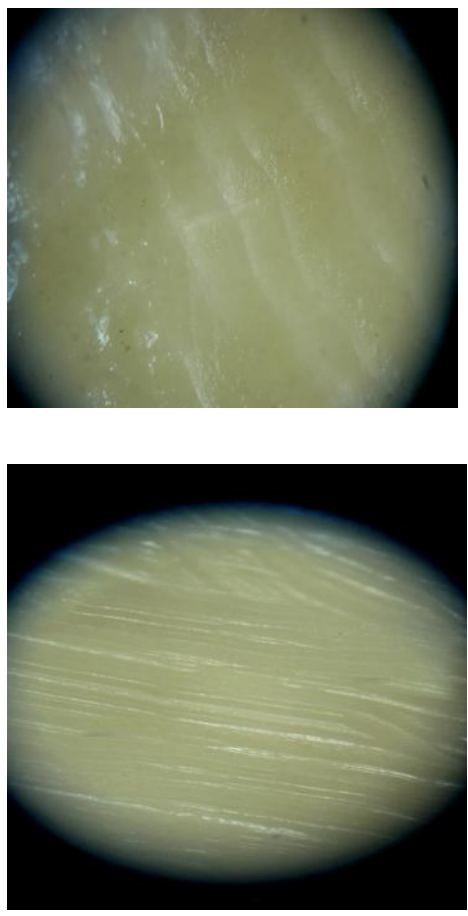

b

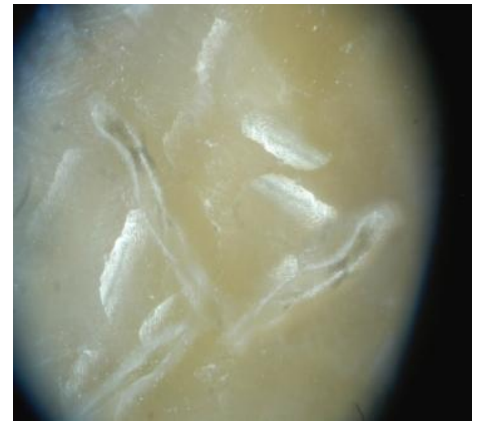

d

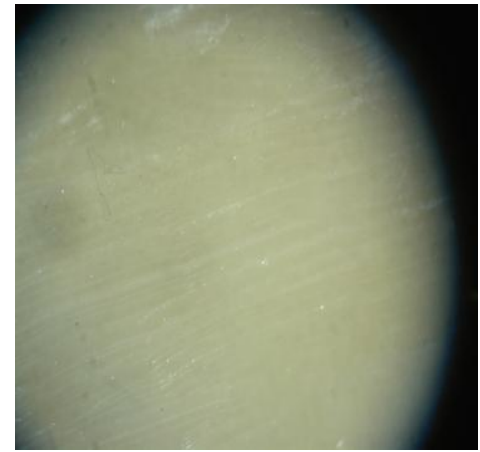

Fig.(2): Stereomicroscopic images of the prepared dentin surfaces.

a: Prepared with diamond round bur. ;b: prepared with carbide round bur.;c: prepared with diamond fissure bur.

d: prepared with carbide fissure bur

a

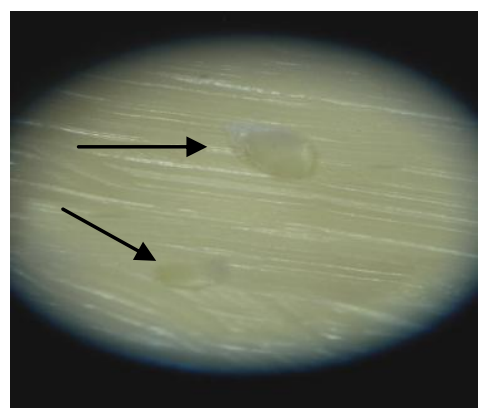

b

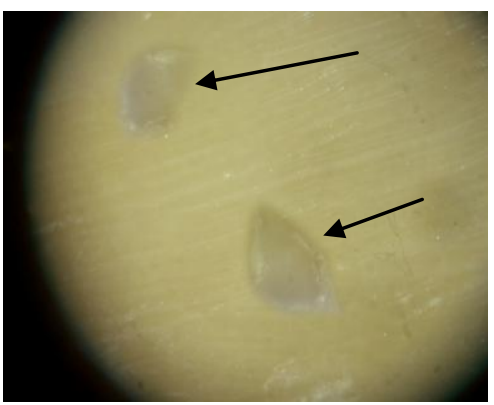

Figure(3): Stereomicroscopic images show the mode of failure $\mathrm{a}:$ cohesive failure when use Excite bonding agent with diamond fissure bur,.b : cohesive failure when use Excite bonding agent with carbide fissure bur.

Table (5): Fracture modes on the specimens after shear bond test in percentages.

\begin{tabular}{ccc}
\hline Group & Adhesive fracture & Mixed \\
\hline G1,I & $90 \%$ & $10 \%$ \\
G2,I & $40 \%$ & $60 \%$ \\
G3,I & $80 \%$ & $20 \%$ \\
G4,I & $10 \%$ & $90 \%$ \\
G1,II & $100 \%$ & $0 \%$ \\
G2,II & $90 \%$ & $10 \%$ \\
G3,II & $100 \%$ & $0 \%$ \\
G4,II & $80 \%$ & $20 \%$ \\
\hline
\end{tabular}




\section{DISCUSSION}

This study evaluated the effect of cutting teeth with different bur types on shear bond strength of composite resin to dentin using total-etch and self-etch adhesive system.

Under the condition of this in vitro study methods of surface preparation greatly affected the shear bond strength of self adhesive systems and this is not true for total etch system, this result agree with the finding of Hosoya et al and Oliveira et $a l^{(1,7)}$ This may due to that self-etch adhesive disclose less etching ability because of their relative high $\mathrm{PH}$ when compared with the $\mathrm{PH}$ of phosphoric acid etchants. ${ }^{(8)}$

The high mean value of shear bond strength obtained with Excite may be due to that the smear layer and super facial dentin are demineralized by $37 \%$ phosphoric acid and the collagen fibers of superficially demineralized dentin are exposed. The exposed collagen may provide reactive groups that can chemically interact with bonding ${ }^{(9)}$. The ethanol solvent of the Excite, due to its high vapor pressure, competes with moisture, replacing it and promoting infiltration of monomer through nano-spaces of the expose collagen network. This serves as a framework for the creation of a resin-demineralized dentin hybrid layer, resulting in strong micromechanical interlocking between resin and the superficially demineralized dentin. ${ }^{(10)}$

The low mean value of shear bond strength of the groups treated with selfetch adhesive system ( I-Bond) related to combine the etching and priming steps, where the primer is not rinsed but only air dried. This result in the calcium and phosphate ions being solubilized from the apitite crystals, which are suspended in the solvents of the primer. When these volatile solvent are evaporated, the concentration of calcium and phosphate may exceed the solubility product constants for calcium and phosphate, resulting in its precipitation within the primer. This result in lesser penetration in dentin. ${ }^{(11,12)}$ In addition to that the hybrid layer formed by self etch adhesive systems thinner than that formed by total etch adhesive systems ${ }^{(13,14)}$

In the self-etch adhesive groups the shear bond strength obtained after dentin preparation with carbide fissure bur higher than that obtained when diamond fissure bur were used. This due to that dentin prepared with diamond burs present a more irregular dentin surface with a visible thicker smear layer when compared to carbide burs treated groups ${ }^{(2,7)}$. The presence of smear layer on dentin surface restrains the effective contact between restorative material and dentinal tissue impairing satisfactory adhesion. ${ }^{(15,16)}$

Fissure burs provide higher shear bond strength than round burs and this may due to the mode of cutting (Figure 3 ), the surface prepared by round bur was rougher than the surface prepared by fissure bur the excessive roughness may impair the even flow of the liquid adhesive and result in an air interrupted at the interface which intern weaken the bond strength. ${ }^{(17,18)}$

The failures were mainly adhesive within the I-Bond self-etching adhesives materials while Excite adhesive (total etch adhesive) show mixed failure and this can be explained by difference in the composition of the bonding agents and the methodology used as stresses within the bonding interface in shear bond test is highly dependent on test geometry and loading configuration employed. This finding is in agreement with Van Noor et al., (1989) and Borges et al., (2006). ${ }^{(19,20)}$

The present study showed that using carbide fissure bur for dentin preparation when using self-etching adhesive is important to improve the shear bond strength of composite resin to dentin. While the method of surface preparation not affected the shear bond strength of composite resin to dentin when using total etch system.

\section{CONCLUSIONS}

Within the limitation of this in vitro study, it could be concluded that:

1-the method of surface preparation affected the shear bond strength of self adhesive systems and this is not true for total etch system.

2- shear bond strength of the groups treated with Excite adhesive (total etch adhesive) have shear bond strength greater than the groups treated with I-Bond ( self 
etch adhesive).

3- when cutting dentin, selection the adequate bur type is important for improved bonding of self-etching adhesive to dentin.

\section{REFERENCES:}

1. Hosoya y, Shinkawa H, Suefiji C, Nozaka $\mathrm{K}$ and Garcia-Godoy. Effect of diamond bur particale size on dentin bond strength. Am J Dent. 2004; 17: 359- 364.

2. Nicol R, Shintome L, Mynaki S and Nagayassu M. Bond strength of resin modified glass ionomer cement to primary dentin after cutting with different bur types and dentin conditioning. $J$ Appl Oral sci. 2007; 15: 459-64.

3. Toledano M, Osorio R, Albaladejo A,Osorio E. Differential effect of in vitro degradation on resin-dentin bonds produced by self etch vs.total-etch adhesive. J Biomed Mat Res A. 2006; 77:128-35.

4. Sturdevants. Art and science of operative dentistry, 4 th ed. Fundamental concepts of enamel and dentin adhesion. St.Louis Mosby; 2002. p. 23768.

5. Spohr A, Correr L,Consani S, Sinhoreti A and Borges G. Effect of refrigeration on tensile bond strength of three adhesive systems. Braz Dent J. 2001; 12:7579.

6. Haak R, Wicht M and Noack M. Dose chemomechanical caries removal affect dentin adhesion? Eur J Oral Sci. 2000; 108:449-455.

7. Oliveira S, Pugach M, Hilton J, Watanabe L, Marshall S, and Marshall G. The influence of the dentin smear layer on adhesion: a self-etching primer vs. a total-etch system. Dent Mat J. 2003; 19: 758-767.

8. Ogata M,Harada N, Yamaguchi S, Nakajima M, Pereira P,Tagami J. Effects of different burs on dentin bond strengths of self-etching primer bonding systems. Oper Dent. 2002; 26:447454.

9. Neelima L, Sathish E and Kandaswamy D. Evaluation of microtensile bond strength of total etch, self etch, and glass ionomer adhesive to human dentin: An in vitro study. Indian J Dent Res. 2008; 19:129-133.

10. Frankenberger R and Karmen N. Dentin bond strength and marginal adaptation. Direct composite resin Vs ceramic inlays. J Oper Dent. 1999; 24:147-155.

11. Demarco F, Tubinol A and Matson E. Tensile bond strength of two dentin adhesive systems. Braz Dent J. 1998; 1: 19-24.

12. Meerrbeek V, Munck J, Yoshida Y, Inoue $\mathrm{S}$, Vargas $\mathrm{M}$ and Vijay P. Adhesion to enamel and dentin: current status and future challenges. Oper Dent. 2002; 6:119-44.

13. Karakaya S, Unlu N, Say E, Ozer F, Soyman $M$ and Tagami J. Bond strength of three different adhesive systems to sclerotic dentin. Dent Mat J. 2008; 27: 471-479.

14. Albaladejo A, Osorio R, Toledano M and Ferrari. Hybrid layer of etch-andrinse versus self etching adhesive systems. Med Oral Path Oral Sur. 2008; 13: 698- 673.

15. Gwinnet J. Smear layer: morphological considerations. Oper Dent. 1984; 9:3-12.

16. Peerzada F. Effect of surface preparation on bond strength of resin luting cements to dentine. 2008; Thesis.

17. Alomari M, Michell $\mathrm{C}$ and Cunningham L. surface roughness and wettability of enamel and dentin surface prepared with different burs. J Oral Rehabil. 2001; 645-650.

18. Rocha P, Borges A, Rodrigues R, Arrais $\mathrm{C}$ and Giannini $\mathrm{M}$. Effect of dental surface preparation on bond strength of self-etching adhesive systems. Braz Dent Res. 2006; 20:52-60.

19. Van Noor R, Noroozi S, Howard IC, Cardew GE. A critique of bond strength measurements. J Dent. 1989; 17:61-67.

20. Borges GA, Spohr AM, Sobrinho LC, Correr AB, Borges LH. Effect of refrigeration on bond strength of self- etching adhesive systems. Braz Dent J. 2006; 17(3): 186-190. 